\title{
Semitensor Product Approach to Controllability, Reachability, and Stabilizability of Probabilistic Finite Automata
}

\author{
Wenhui Dou $\left(\mathbb{D},{ }^{1}\right.$ Haitao Li $\left(\mathbb{D},{ }^{1}\right.$ and Fuad E. Alsaadi ${ }^{2}{ }^{2}$ \\ ${ }^{1}$ School of Mathematics and Statistics, Shandong Normal University, Jinan 250014, China \\ ${ }^{2}$ Department of Electrical and Computer Engineering, Faculty of Engineering, King Abdulaziz University, Jeddah 21589, Saudi Arabia \\ Correspondence should be addressed to Haitao Li; haitaoli09@gmail.com
}

Received 5 December 2018; Accepted 1 April 2019; Published 14 April 2019

Academic Editor: Emilio Jiménez Macías

Copyright (C) 2019 Wenhui Dou et al. This is an open access article distributed under the Creative Commons Attribution License, which permits unrestricted use, distribution, and reproduction in any medium, provided the original work is properly cited.

\begin{abstract}
This paper proposes a matrix-based approach to investigate the controllability, reachability, and stabilizability of probabilistic finite automata (PFA). Firstly, the state transition probabilistic structure matrix is constructed for PFA, based on which a kind of controllability matrix is defined for PFA. Secondly, some necessary and sufficient conditions are presented for the controllability, reachability, and stabilizability of PFA with positive probability by using the controllability matrix. Finally, an illustrate example is given to validate the obtained new results.
\end{abstract}

\section{Introduction}

Finite automaton is a kind of finite-state machine which plays a key role in theoretical computer science. There are many kinds of finite automata, such as deterministic finite automata (DFA) $[1,2]$, nondeterministic finite automata (NFA) [3], probabilistic finite automata (PFA) [4, 5], and so on. In the past few decades, finite automata has attracted lots of scholars' research interest in many disciplines such as computer science, applied mathematics, and engineering, and some fundamental results have been presented $[6,7]$.

Especially, finite automaton is an important model of discrete event systems (DESs). Another effective model of DESs is Petri net. As we all know, controllability, reachability, and observability are fundamental concepts in DESs [8-10]. The concepts of controllability and observability are extensively studied in Petri nets [11-14], while the concept of reachability is intrinsic to both finite automata $[1,2]$ and Petri nets [15]. In finite automata, the concept of reachability comes from the classic control theory, which depends on the occurrence events of every state $[1,2]$. However, in bounded Petri nets, the concept of reachability depends on transitions and tokens of every marking, which is quite different from that of finite automata [15].

Recently, PFA has been extensively studied because it generalizes the concept of NFA by adding transition probability to the transition function and has wider applications [16-18]. The state estimation and detectability of PFA were considered in $[4,19]$, and some necessary and sufficient conditions were presented. The supervisory control of PFA was investigated in [20], and several supervisor synthesis problems were proposed.

In some recent works, a semitensor product (STP) approach [21] has been introduced to the reachability and stabilizability of finite automata $[1,2,22]$. For other applications of STP in Boolean networks [23-25] and game theory [2628], please refer to [29-35]. Xu et al. [1] developed an STPbased method for the reachability of DFA. Yan et al. [2] presented some new criteria for the controllability, reachability and stabilizability of DFA by using STP. Zhang et al. [18] established the algebraic form of PFA via STP and studied the reachability of PFA with positive probability and with probability one, respectively. However, to our best knowledge, there are fewer results on the analysis of controllability and stabilizability of PFA.

In this paper, we propose a matrix-based approach to investigate the controllability, reachability, and stabilizability of PFA with positive probability. The main contributions of this paper are twofold. On one hand, we construct the controllability matrix for PFA, which is effective for the analysis of PFA. On the other hand, some necessary and sufficient conditions are obtained for the controllability, 
reachability, and stabilizability of PFA with positive probability. These conditions are based on the controllability matrix, and easily verified via MATLAB. Compared with the existing results [18], this paper introduces the concept of controllability and stabilizability for PFA with positive probability.

The rest of this paper is organized as follows. Section 2 introduces some necessary preliminaries on PFA. Section 3 presents some necessary and sufficient conditions for the controllability, reachability, and stabilizability of PFA with positive probability. In Section 4, an example is given to illustrate the new results, which is followed by a brief conclusion in Section 5.

Notations. $M_{i, j}$ is the $(i, j)$-th element of matrix $M . \mathbf{0}_{n}=$ $\left[\begin{array}{llll}\underbrace{0}_{n} & \cdots & \cdots\end{array}\right] \in \mathbb{R}^{1 \times n} \cdot \delta_{n}^{i}$ represents the $i$-th column of the identity matrix $I_{n} . \Delta_{n}:=\left\{\delta_{n}^{1}, \cdots, \delta_{n}^{n}\right\}$. The matrix product of this paper is the semitensor product of matrices [21]. We often omit the symbol " $\propto$ " if no confusion arises.

\section{Preliminaries on PFA}

A probabilistic finite automata is a five-tuple $\Lambda=$ $\left(X, \Sigma, f, x^{0}, p\right)$, where $X=\left\{x_{1}, x_{2}, \cdots, x_{n}\right\}$ is the finite set of states, $x^{0} \in X$ is the initial state, $\Sigma=\left\{\sigma_{1}, \cdots, \sigma_{m}\right\}$ is the finite set of events called alphabet, $\Sigma^{*}$ is the finite input string set on $\Sigma$, and $f: X \times \Sigma \longrightarrow 2^{X}$ is the partial transition function. Given a state $x_{p} \in X$ and a finite input string $s=\sigma^{(0)} \sigma^{(1)} \cdots \sigma^{(t-1)} \in \Sigma^{*}$, we define $f\left(x_{p}, s\right)=f\left(\cdots f\left(f\left(x_{p}, \sigma^{(0)}\right), \sigma^{(1)}\right), \cdots, \sigma^{(t-1)}\right)$. In this paper, we assume that, for any state $x \in X$, there exists an event $\sigma \in \Sigma$ such that $f(x, \sigma) \in X . p: X \times \Sigma \times X \longrightarrow[0,1]$ is the state transition probabilistic function, that is, $p\left(x_{p}, \sigma, x_{q}\right)$ means the probability from state $x_{p}$ to state $x_{q}$ with the occurrence of event $\sigma$. We notice that $\sum_{\sigma \in \Sigma} \sum_{x_{q} \in X} p\left(x_{p}, \sigma, x_{q}\right)=1$ holds for any $x_{p} \in X$.

Now, we present the concepts of controllability, reachability, and stabilizability for PFA.

Definition 1. (1) The state $x_{\alpha} \in X$ is said to be controllable to the state $x_{\beta} \in X$ with positive probability, if there exists a finite input sequence $s \in \Sigma^{*},|s| \geq 1$, such that $p\left(x_{\alpha}, s, x_{\beta}\right)>$ 0 . Here, $|s|$ means the length of finite input sequence s.

(2) The state $x_{\alpha} \in X$ is said to be controllable with positive probability, if the state $x_{\alpha} \in X$ is controllable to any state $x_{\beta} \in$ $X$ with positive probability.

Definition 2. (1) The state $x_{\beta} \in X$ is said to be reachable from the state $x_{\alpha} \in X$ with positive probability, if there exists a finite input sequence $s \in \Sigma^{*},|s| \geq 1$, such that $p\left(x_{\alpha}, s, x_{\beta}\right)>$ 0 .

(2) The state $x_{\beta} \in X$ is said to be reachable with positive probability, if the state $x_{\beta} \in X$ is reachable from any state $x_{\alpha} \in X$ with positive probability.

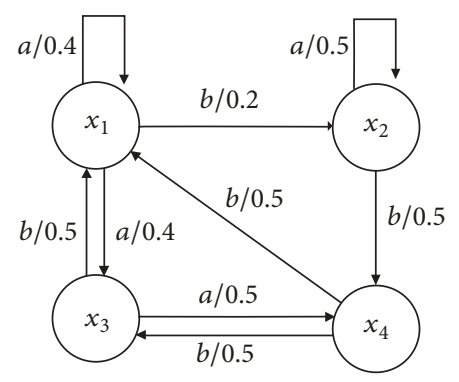

FIGURE 1: A probabilistic finite automata.

Given two nonempty sets $X_{1} \subseteq X$ and $X_{2} \subseteq X$, we assume that

$$
\begin{aligned}
& X_{1} \cup X_{2}=X, \\
& X_{1} \cap X_{2}=\emptyset .
\end{aligned}
$$

Then, we give the concepts of controllability, reachability and stabilizability of nonempty sets of states.

Definition 3. The nonempty set of states $X_{1} \subseteq X$ is said to be controllable with positive probability, if for any state $x_{\beta} \in X_{2}$, there exists a state $x_{\alpha} \in X_{1}$ such that $x_{\alpha}$ is controllable to $x_{\beta}$ with positive probability.

Definition 4. The nonempty set of states $X_{2} \subseteq X$ is said to be reachable with positive probability, if for any state $x_{\alpha} \in X_{1}$, there exists a state $x_{\beta} \in X_{2}$ such that $x_{\beta}$ is reachable from $x_{\alpha}$ with positive probability.

Definition 5. The nonempty set of states $X_{1} \subseteq X$ is said to be one-step returnable with positive probability, if for any state $x_{\alpha} \in X_{1}$, there exist an event $\sigma \in \Sigma$ and a state $x_{\beta} \in X_{1}$ such that $p\left(x_{\alpha}, \sigma, x_{\beta}\right)>0$.

Definition 6. The nonempty set of states $X_{1} \subseteq X$ is said to be stabilizable with positive probability, if $X_{1}$ is reachable with positive probability and one-step returnable with positive probability.

Example 7. Consider a PFA $\Lambda=\left(X, \Sigma, f, x^{0}, p\right)$, where $X=$ $\left\{x_{1}, x_{2}, x_{3}, x_{4}\right\}, \Sigma=\{a, b\}, x^{0}=x_{1}$, and $f$ and $p$ are shown in Figure 1 .

From Figure 1, we can see that $x_{1} \stackrel{b / 0.2}{\longrightarrow} x_{2} \stackrel{b / 0.5}{\longrightarrow} x_{4} \stackrel{b / 0.5}{\longrightarrow}$ $x_{3} \stackrel{b / 0.5}{\longrightarrow} x_{1}$. Therefore, by Definitions 1 and $2, x_{1}, x_{2}, x_{3}$, and $x_{4}$ are controllable and reachable with positive probability.

Assume that $X_{1}=\left\{x_{1}, x_{2}\right\}$ and $X_{2}=\left\{x_{3}, x_{4}\right\}$. Since $x_{1} \in X_{1} \stackrel{b / 0.2}{\longrightarrow} x_{2} \stackrel{b / 0.5}{\longrightarrow} x_{4} \in X_{2}$ and $x_{1} \in X_{1} \stackrel{a / 0.4}{\longrightarrow} x_{3} \in X_{2}$, by Definition $3, X_{1}$ is controllable with positive probability. Similarly, $X_{2}$ is also controllable with positive probability. Since $x_{3} \stackrel{a / 0.5}{\longrightarrow} x_{4} \stackrel{b / 0.5}{\longrightarrow} x_{1} \stackrel{b / 0.2}{\longrightarrow} x_{2}$ and $x_{1} \stackrel{b / 0.2}{\longrightarrow} x_{2} \stackrel{b / 0.5}{\longrightarrow}$ $x_{4} \stackrel{b / 0.5}{\longrightarrow} x_{3}$, by Definition 4 , we can obtain that $X_{1}$ and $X_{2}$ are reachable with positive probability. Since $x_{3} \stackrel{a / 0.5}{\longrightarrow} x_{4}$ and 
$x_{4} \stackrel{b / 0.5}{\longrightarrow} x_{3}$, by Definition $5, X_{2}$ is one-step returnable with positive probability. Hence, by Definition $6, X_{2}$ is stabilizable with positive probability.

\section{Main Results}

In this section, we give a controllability matrix to investigate the probability finite automata (PFA) about its controllability, reachability, and stabilizability.

Given a PFA $\Lambda=\left(X, \Sigma, f, x^{0}, p\right)$, we identify $x_{\alpha} \sim \delta_{n}^{\alpha}, \alpha=$ $1, \cdots, n, \sigma_{t} \sim \delta_{m}^{t}, t=1, \cdots, m$, and $\left(P_{t}\right)_{\beta, \alpha}:=p\left(x_{\alpha}, \sigma_{t}, x_{\beta}\right)$.

We construct the state transition probabilistic structure matrix (STPSM) of $\Lambda$ as

$$
L=\left[L_{1} \cdots L_{m}\right] \in \mathbb{R}^{n \times m n}
$$

where $L_{t} \in \mathbb{R}^{n \times n}$ is defined as follows:

$$
\left(L_{t}\right)_{\beta, \alpha}:= \begin{cases}\left(P_{t}\right)_{\beta, \alpha}, & \text { if } \delta_{n}^{\beta} \in f\left(\delta_{n}^{\alpha}, \delta_{m}^{t}\right), \\ 0, & \text { otherwise. }\end{cases}
$$

Based on the construction of $L$, the considered PFA has the following algebraic form:

$$
E x(t+1)=L u(t) E x(t)
$$

where $E x(t)$ is the mathematic expectation of states at time $t$, $u(t) \in \Delta_{m}$ is the vector of events at time $t$, and $L$ is the STPSM.

For any positive integer $t$, let

$$
K:=\overbrace{\left[\begin{array}{ccccc}
1 & 1 & \cdots & 1 & 1 \\
1 & 1 & \cdots & 1 & 2 \\
\vdots & \vdots & \vdots & \vdots & \vdots \\
1 & 1 & \cdots & 1 & m \\
1 & 1 & \cdots & 2 & 1 \\
\vdots & \vdots & \vdots & \vdots & \vdots \\
m & m & \cdots & m & m-1 \\
m & m & \cdots & m & m
\end{array}\right]}^{t} \in \mathbb{R}^{m^{t} \times t} .
$$

Then, the $i$-th row of matrix $K$, denoted by $\left(K_{i, 1}, \cdots, K_{i, t}\right)$, represents the choice of $\left\{L_{K_{i, 1}}, \cdots, L_{K_{i, t}}\right\}$ in $\left\{L_{1} L_{2} \cdots L_{m}\right\}$.

Define

$$
\mathscr{M}^{(t)}=\left\{H_{1}^{(t)}, H_{2}^{(t)}, \cdots, H_{m^{t}}^{(t)}\right\}
$$

where $H_{i}^{(t)}=\prod_{j=1}^{t} L_{K_{i, t-j+1}}$. Then, $\mathscr{M}^{(t)}$ denotes the set of $t$-step transition probability matrices associated with all possible inputs with length $t$.
Given a finite input sequence $s=\sigma^{(0)} \sigma^{(1)} \cdots \sigma^{(t-1)} \in \Sigma^{*}$ with $s \sim \delta_{m^{t}}^{r}$, by (4) and (6), we have

$$
\begin{aligned}
E x(t) & =L \sigma^{(t-1)} E x(t-1)=L_{K_{r, t}} L \sigma^{(t-2)} E x(t-2) \\
& =L_{K_{r, t}} L_{K_{r, t-1}} L \sigma^{(t-3)} E x(t-3)=\cdots \\
& =L_{K_{r, t}} L_{K_{r, t-1}} \cdots \sigma^{(0)} x(0) \\
& =L_{K_{r, t}} L_{K_{r, t-1}} \cdots L_{K_{r, 1}} x(0)=H_{r}^{(t)} x(0) .
\end{aligned}
$$

Set

$$
M_{t}=\sum_{i=1}^{m^{t}} H_{i}^{(t)} \in \mathbb{R}^{n \times n} .
$$

Comparing (6) with $\left(\sum_{i=1}^{m} L_{i}\right)^{t}$, one can find that

$$
M_{t}=\left(\sum_{i=1}^{m} L_{i}\right)^{t} \in \mathbb{R}^{n \times n} .
$$

Theorem 8. (1) The state $x_{\alpha}$ is controllable to the state $x_{\beta}$ with positive probability, if and only if there exists a positive integer $\tau$ such that $(C)_{\beta, \alpha}>0$, where

$$
C=\sum_{t=1}^{\tau} M_{t}
$$

(2) The state $x_{\alpha}$ is controllable with positive probability, if and only if there exists a positive integer $\tau$ such that

$$
\operatorname{Col}_{\alpha}(C)=\operatorname{Col}_{\alpha}\left(\sum_{t=1}^{\tau} M_{t}\right)>\mathbf{0}_{n}^{\top}
$$

Proof. We firstly prove conclusion (1).

(Necessity) Suppose that the state $x_{\alpha}$ is controllable to the state $x_{\beta}$ with positive probability. By Definition 1 , there exists a finite input sequence $s=\sigma^{(0)} \sigma^{(1)} \cdots \sigma^{(\tau-1)} \in \Sigma^{*}$ such that $p\left(x_{\alpha}, s, x_{\beta}\right)>0$. We assume that $s \sim \delta_{m^{\tau}}^{r}$. By (7), one can see that $\left(H_{r}^{(\tau)}\right)_{\beta, \alpha}>0$, which shows that

$$
\left(M_{\tau}\right)_{\beta, \alpha}=\sum_{i=1}^{m^{\tau}}\left(H_{i}^{(\tau)}\right)_{\beta, \alpha} \geq\left(H_{r}^{(\tau)}\right)_{\beta, \alpha}>0 .
$$

Therefore, there exists a positive integer $\tau$ such that $(C)_{\beta, \alpha}=$ $\sum_{t=1}^{\tau}\left(M_{t}\right)_{\beta, \alpha} \geq\left(M_{\tau}\right)_{\beta, \alpha}>0$ holds.

(Sufficiency) Suppose that there exists a positive integer $\tau$ such that $(C)_{\beta, \alpha}=\sum_{t=1}^{\tau}\left(M_{t}\right)_{\beta, \alpha}>0$. Then, there exists a positive integer $\lambda \leq \tau$ such that $\left(M_{\lambda}\right)_{\beta, \alpha}>0$. Since $\left(M_{\lambda}\right)_{\beta, \alpha}=$ $\sum_{i=1}^{m^{\lambda}}\left(H_{i}^{(\lambda)}\right)_{\beta, \alpha}>0$, there exists a positive integer $r \leq m^{\lambda}$ such that $\left(H_{r}^{(\lambda)}\right)_{\beta, \alpha}>0$. By (7), one can find a finite input sequence $s=\sigma^{(0)} \sigma^{(1)} \cdots \sigma^{(\lambda-1)} \in \Sigma^{*}$ with $s \sim \delta_{m^{\lambda}}^{r}$, such that 
$p\left(x_{\alpha}, s, x_{\beta}\right)=\left(H_{r}^{(\lambda)}\right)_{\beta, \alpha}>0$. By Definition 1, $x_{\alpha}$ is controllable to $x_{\beta}$ with positive probability.

In the following, we prove conclusion (2).

(Necessity) Suppose that the state $x_{\alpha} \sim \delta_{n}^{\alpha}$ is controllable with positive probability. By Definition 1 , for any state $x_{\beta} \sim$ $\delta_{n}^{\beta}, \delta_{n}^{\alpha}$ is controllable to $\delta_{n}^{\beta}$ with positive probability. Then, by conclusion (1) of Theorem 8 , there exists a positive integer $\tau_{\beta}$ such that

$$
\sum_{t=1}^{\tau_{\beta}}\left(M_{t}\right)_{\beta, \alpha}>0
$$

Let $\tau=\max \left\{\tau_{\beta}: \beta=1,2, \cdots, n\right\}$. Then, one can obtain that

$$
\operatorname{Col}_{\alpha}\left(\sum_{t=1}^{\tau} M_{t}\right)>\mathbf{0}_{n}^{\top}
$$

(Sufficiency) Suppose that there exists a positive integer $\tau$ such that (11) holds. Then for any $\beta=1, \cdots, n$, we have $\sum_{t=1}^{\tau}\left(M_{t}\right)_{\beta, \alpha}>0$. By conclusion (1) of Theorem 8 , the state $x_{\alpha}$ is controllable to the state $x_{\beta}$ with positive probability. Since $\beta$ is arbitrary, the state $x_{\alpha} \in X$ is controllable to any state $x_{\beta} \in X$ with positive probability. By Definition 1, the state $x_{\alpha} \in X$ is controllable with positive probability.

Theorem 9. (1) The state $x_{\beta}$ is reachable from the state $x_{\alpha}$ with positive probability, if and only if there exists a positive integer $\tau$ such that $(C)_{\beta, \alpha}>0$.

(2) The state $x_{\beta}$ is reachable with positive probability, if and only if there exists a positive integer $\tau$ such that

$$
\operatorname{Row}_{\beta}(C)=\operatorname{Row}_{\beta}\left(\sum_{t=1}^{\tau} M_{t}\right)>\mathbf{0}_{n} .
$$

Proof. The proof of conclusion (1) is very similar to that of Theorem 8. We just prove conclusion (2).

(Necessity) Suppose that the state $x_{\beta} \sim \delta_{n}^{\beta}$ is reachable with positive probability. By Definition 2 , for any state $x_{\alpha} \sim$ $\delta_{n}^{\alpha}, \delta_{n}^{\beta}$ is reachable from $\delta_{n}^{\alpha}$ with positive probability. From conclusion (1) of Theorem 9, there exists a positive integer $\tau_{\alpha}$ such that

$$
\sum_{t=1}^{\tau_{\alpha}}\left(M_{t}\right)_{\beta, \alpha}>0
$$

Set $\tau=\max \left\{\tau_{\alpha}: \alpha=1,2, \cdots, n\right\}$. It is easy to see that (15) holds.

(Sufficiency) Suppose that there exists a positive integer $\tau$ such that (15) holds. Then for any $\alpha=1, \cdots, n$, we have $\sum_{t=1}^{\tau}\left(M_{t}\right)_{\beta, \alpha}>0$. By conclusion (1) of Theorem 9, the state $x_{\beta}$ is reachable from the state $x_{\alpha}$ with positive probability. Since $\alpha$ is arbitrary, one can obtain that the state $x_{\beta} \in X$ is reachable from any state $x_{\alpha} \in \mathrm{X}$ with positive probability. By Definition 2, the state $x_{\beta} \in X$ is reachable with positive probability.
Given the two nonempty sets of states

$$
\begin{aligned}
& X_{1}=\left\{\delta_{n}^{\alpha_{1}}, \delta_{n}^{\alpha_{2}}, \cdots, \delta_{n}^{\alpha_{l}}\right\}, \\
& X_{2}=\left\{\delta_{n}^{\alpha_{l+1}}, \delta_{n}^{\alpha_{l+2}}, \cdots, \delta_{n}^{\alpha_{n}}\right\},
\end{aligned}
$$

it is obvious that $X_{1} \cup X_{2}=X$ and $X_{1} \cap X_{2}=\emptyset$.

According to (9) and (10), we define the following matrices:

$$
\begin{aligned}
C_{X_{1}} & =\left[\sum_{j=1}^{l}(C)_{\alpha_{l+1}, \alpha_{j}} \sum_{j=1}^{l}(C)_{\alpha_{l+2}, \alpha_{j}} \cdots \sum_{j=1}^{l}(C)_{\alpha_{n}, \alpha_{j}}\right], \\
R_{X_{2}} & =\left[\sum_{i=l+1}^{n}(C)_{\alpha_{i}, \alpha_{1}} \sum_{i=l+1}^{n}(C)_{\alpha_{i}, \alpha_{2}} \cdots \sum_{i=l+1}^{n}(C)_{\alpha_{i}, \alpha_{l}}\right], \\
M_{X_{1}} & =\left[\sum_{i=1}^{l}(M)_{\alpha_{i}, \alpha_{1}} \sum_{i=1}^{l}(M)_{\alpha_{i}, \alpha_{2}} \cdots \sum_{i=1}^{l}(M)_{\alpha_{i}, \alpha_{l}}\right] .
\end{aligned}
$$

Theorem 10. $X_{1} \subseteq X$ is controllable with positive probability, if and only if $C_{X_{1}}>\mathbf{0}_{n-l}$.

Proof. (Necessity) Suppose that $X_{1} \subseteq X$ is controllable with positive probability. By Definition 3, for any state $\delta_{n}^{\alpha_{i}} \in X_{2}, i=$ $l+1, \cdots, n$, there exist a state $\delta_{n}^{\alpha_{j_{i}}} \in X_{1}$ and a control sequence $s_{i} \in \Sigma^{*}$ such that $p\left(\delta_{n}^{\alpha_{j_{i}}}, s_{i}, \delta_{n}^{\alpha_{i}}\right)>0$. Based on conclusion (1) of Theorem 8 , one can obtain $(C)_{\alpha_{i}, \alpha_{j_{i}}}>0, i=l+1, \cdots, n$. Therefore,

$$
\sum_{j=1}^{l}(C)_{\alpha_{i}, \alpha_{j}} \geq(C)_{\alpha_{i}, \alpha_{j_{i}}}>0, \quad \forall i=l+1, \cdots, n,
$$

which shows that $C_{X_{1}}>\mathbf{0}_{n-l}$.

(Sufficiency) Assume that $C_{X_{1}}>\mathbf{0}_{n-l}$. Then for any $i=$ $l+1, \cdots, n$, we have $\sum_{j=1}^{l}(C)_{\alpha_{i}, \alpha_{j}}>0$. Thus, for any state $\delta_{n}^{\alpha_{i}} \in X_{2}, i=l+1, \cdots, n$, there exist $\delta_{n}^{\alpha_{j_{i}}} \in X_{1}$ and a control sequence $s_{i} \in \Sigma^{*}$ such that $p\left(\delta_{n}^{\alpha_{j_{i}}}, s_{i}, \delta_{n}^{\alpha_{i}}\right)>0$. By Definition 3, $X_{1} \subseteq X$ is controllable with positive probability.

Theorem 11. $X_{2} \subseteq X$ is reachable with positive probability, if and only if $R_{X_{2}}>\mathbf{0}_{l}$.

Proof. (Necessity) Suppose that $X_{2} \subseteq X$ is reachable with positive probability. By Definition 4 , for any state $\delta_{n}^{\alpha_{j}} \in X_{1}$, $j=1, \cdots, l$, there exist a state $\delta_{n}^{\alpha_{i_{j}}} \in X_{2}$ and a control sequence $s_{j} \in \Sigma^{*}$ such that $p\left(\delta_{n}^{\alpha_{j}}, s_{j}, \delta_{n}^{\alpha_{i_{j}}}\right)>0$, which together with conclusion (1) of Theorem 9 implies that $(C)_{\alpha_{i j}, \alpha_{j}}>0$, $j=1, \cdots, l$. Hence,

$$
\sum_{i=l+1}^{n}(C)_{\alpha_{i}, \alpha_{j}} \geq(C)_{\alpha_{i_{j}}, \alpha_{j}}>0, \quad \forall j=1, \cdots, l .
$$

From the arbitrariness of $\alpha_{j}$, one can see that $R_{X_{2}}>\mathbf{0}_{l}$.

(Sufficiency) Assume that $R_{X_{2}}>\mathbf{0}_{l}$. Then for any $j=$ $1, \cdots, l$, we have $\sum_{i=l+1}^{n}(C)_{\alpha_{i}, \alpha_{j}}>0$. Hence, for any state 


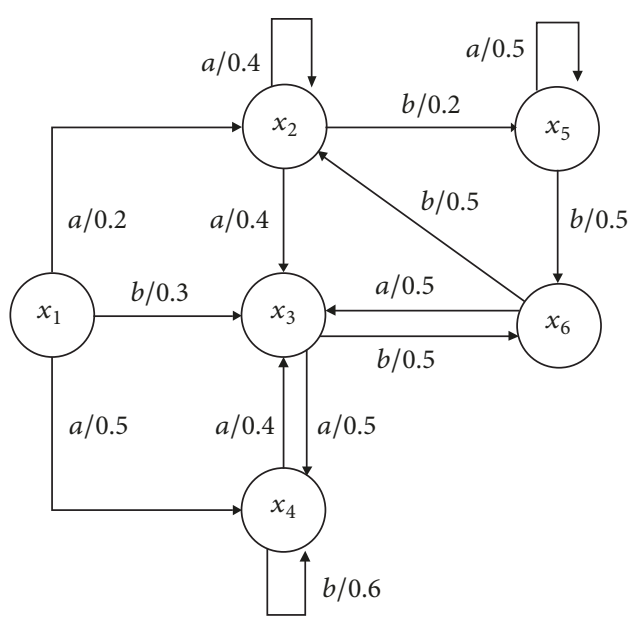

FIgURE 2: A probabilistic finite automata.

$\delta_{n}^{\alpha_{j}} \in X_{1}$, there exist $\delta_{n}^{\alpha_{i_{j}}} \in X_{2}$ and a control sequence $s_{j} \in \Sigma^{*}$ such that $p\left(\delta_{n}^{\alpha_{j}}, s_{j}, \delta_{n}^{\alpha_{i j}}\right)>0$. By Definition $4, X_{2} \subseteq X$ is reachable with positive probability.

Theorem 12. $X_{1} \subseteq X$ is one-step returnable with positive probability, if and only if $M_{X_{1}}>\mathbf{0}_{l}$.

Proof. (Necessity) Suppose that $X_{1} \subseteq X$ is one-step returnable with positive probability. By Definition 5, for any state $\delta_{n}^{\alpha_{j}} \in X_{1}$, there exist a state $\delta_{n}^{\alpha_{i_{j}}} \in X_{1}$ and an event $\sigma_{j} \in \Sigma$ such that $p\left(\delta_{n}^{\alpha_{j}}, \sigma_{j}, \delta_{n}^{\alpha_{i_{j}}}\right)>0$. Therefore,

$$
(M)_{\alpha_{i j}, \alpha_{j}}>0, \quad \forall j=1, \cdots, l
$$

which implies that $\sum_{i=1}^{l}(M)_{\alpha_{i}, \alpha_{j}} \geq(M)_{\alpha_{i_{j}}, \alpha_{j}}>0$. Thus, $M_{X_{1}}>$ $\mathbf{0}_{l}$.

(Sufficiency) Assume that $M_{X_{1}}>\mathbf{0}_{l}$. Then for any $j=$ $1, \cdots, l$, we have $\sum_{i=1}^{l}(M)_{\alpha_{i}, \alpha_{j}}>0$. Hence, for any state $\delta_{n}^{\alpha_{j}} \in$ $X_{1}$, there exist $\delta_{n}^{\alpha_{i_{j}}} \in X_{1}$ and a control sequence $\sigma_{j} \in \Sigma$ such that $p\left(\delta_{n}^{\alpha_{j}}, \sigma_{j}, \delta_{n}^{\alpha_{i_{j}}}\right)>0$. By Definition $5, X_{1} \subseteq X$ is one-step returnable with positive probability.

Based on Definition 6, Theorems 11 and 12, we have the following result on the stabilizability of PFA.

Theorem 13. $X_{1} \subseteq X$ is stabilizable with positive probability, if and only if $R_{X_{1}}>\mathbf{0}_{n-l}$ and $M_{X_{1}}>\mathbf{0}_{l}$.

\section{An Illustrate Example}

In this section, we present an example to illustrate the main results.

Example 14. Consider a PFA $\Lambda=\left(X, \Sigma, f, x^{0}, p\right)$ shown in Figure 2, where $X=\left\{x_{1}, x_{2}, x_{3}, x_{4}, x_{5}, x_{6}\right\}, \Sigma=\{a, b\}$ and $x^{0}=x_{1}$. Assume that $X_{1}=\left\{x_{1}, x_{2}\right\}$ and $X_{2}=\left\{x_{3}, x_{4}, x_{5}, x_{6}\right\}$.
Identify $X \sim \Delta_{6}=\left\{\delta_{6}^{1}, \delta_{6}^{2}, \cdots, \delta_{6}^{6}\right\}$ and $\Sigma \sim \Delta_{2}=\left\{\delta_{2}^{1}, \delta_{2}^{2}\right\}$. The state transition probabilistic structure matrix of PFA $\Lambda$ shown in Figure 2 is

$$
\begin{aligned}
L & =\left[\begin{array}{ll}
L_{1} & L_{2}
\end{array}\right] \\
& =\left[\begin{array}{cccccccccccc}
0 & 0 & 0 & 0 & 0 & 0 & 0 & 0 & 0 & 0 & 0 & 0 \\
0.2 & 0.4 & 0 & 0 & 0 & 0 & 0 & 0 & 0 & 0 & 0 & 0.5 \\
0 & 0.4 & 0 & 0.4 & 0 & 0.5 & 0.3 & 0 & 0 & 0 & 0 & 0 \\
0.5 & 0 & 0.5 & 0 & 0 & 0 & 0 & 0 & 0 & 0.6 & 0 & 0 \\
0 & 0 & 0 & 0 & 0.5 & 0 & 0 & 0.2 & 0 & 0 & 0 & 0 \\
0 & 0 & 0 & 0 & 0 & 0 & 0 & 0 & 0.5 & 0 & 0.5 & 0
\end{array}\right],
\end{aligned}
$$

where $L_{1}=\left[\begin{array}{cccccc}0 & 0 & 0 & 0 & 0 & 0 \\ 0.2 & 0.4 & 0 & 0 & 0 & 0 \\ 0 & 0.4 & 0 & 0.4 & 0 & 0.5 \\ 0.5 & 0 & 0.5 & 0 & 0 & 0 \\ 0 & 0 & 0 & 0 & 0.5 & 0 \\ 0 & 0 & 0 & 0 & 0 & 0\end{array}\right]$

$L_{2}=\left[\begin{array}{cccccc}0 & 0 & 0 & 0 & 0 & 0 \\ 0 & 0 & 0 & 0 & 0 & 0.5 \\ 0.3 & 0 & 0 & 0 & 0 & 0 \\ 0 & 0 & 0 & 0.6 & 0 & 0 \\ 0 & 0.2 & 0 & 0 & 0 & 0 \\ 0 & 0 & 0.5 & 0 & 0.5 & 0\end{array}\right]$

Set $\tau=4$. Then

$C=\sum_{t=1}^{4} M_{t}$

$$
=\left[\begin{array}{cccccc}
0 & 0 & 0 & 0 & 0 & 0 \\
0.5098 & 0.9446 & 0.5025 & 0.2 & 0.69 & 1.062 \\
1.1538 & 1.1046 & 0.9845 & 1.2464 & 0.74 & 1.262 \\
1.7495 & 0.667 & 1.558 & 2.0516 & 0.3125 & 0.8025 \\
0.1154 & 0.6058 & 0.095 & 0.02 & 1.0575 & 0.276 \\
0.4715 & 0.678 & 0.86 & 0.482 & 1.2 & 0.5975
\end{array}\right] .
$$

By Theorem 8 , the state $x^{0}=x_{1}$ is controllable to the any state except the state $x_{1}$ with positive probability.

A simple calculation shows that $C_{X_{1}}=[2.25842 .4165$ 0.72121 .1495 ], $R_{X_{1}}=\left[\begin{array}{llll}0.5025 & 0.2 & 0.69 & 1.062\end{array}\right], C_{X_{2}}=$ [0 2.4545], $R_{X_{2}}=\left[\begin{array}{lll}3.4902 & 3.0554\end{array}\right], M_{X_{1}}=\left[\begin{array}{lll}0.5098 & 0.9446\end{array}\right]$, and $M_{X_{2}}=\left[\begin{array}{llll}3.4975 & 3.8 & 3.31 & 2.938\end{array}\right]$. By Theorem $10, X_{1}$ is controllable with positive probability and $X_{2}$ is not controllable with positive probability. By Theorems 11, 12, and 13 , both $X_{1}$ and $X_{2}$ are reachable, one-step returnable, and stabilizable with positive probability, respectively.

\section{Conclusion}

In this paper, we have proposed a controllability matrix approach for the investigation of PFA via STP. Based on the controllability matrix, we have presented some necessary and 
sufficient conditions for the controllability, reachability, and stabilizability of PFA with positive probability. The obtained conditions are easily verified via MATLAB.

\section{Data Availability}

No data were used to support this study.

\section{Conflicts of Interest}

The authors declare that there are no conflicts of interest regarding the publication of this paper.

\section{Acknowledgments}

The research was supported by the National Natural Science Foundation of China under Grants 61873150 and 61503225 and the Natural Science Fund for Distinguished Young Scholars of Shandong Province under Grant JQ201613.

\section{References}

[1] X. Xu and Y. Hong, "Matrix expression and reachability analysis of finite automata," Control Theory and Technology, vol. 10, no. 2, pp. 210-215, 2012.

[2] Y. Yan, Z. Chen, and Z. Liu, "Semi-tensor product approach to controllability and stabilizability of finite automata," Journal of Systems Engineering and Electronics, vol. 26, no. 1, pp. 134-141, 2015.

[3] V. Geffert, C. Mereghetti, and G. Pighizzini, "Converting twoway nondeterministic unary automata into simpler automata," Theoretical Computer Science, vol. 295, no. 1-3, pp. 189-203, 2003.

[4] C. Keroglou and C. N. Hadjicostis, "Verification of detectability in probabilistic finite automata," Automatica, vol. 86, pp. 192198, 2017.

[5] Y. Wen and A. Ray, "Vector space formulation of probabilistic finite state automata," Journal of Computer and System Sciences, vol. 78, no. 4, pp. 1127-1141, 2012.

[6] C. G. Cassandras and S. Lafortune, Introduction to Discrete Event Systems, Kluwer Academic Publishers, Dordrecht, Netherlands, 1999.

[7] R. Kumar and V. K. Garg, Modeling and Control of Logical Discrete Event Systems, Kluwer Academic Publishers, Dordrecht, Netherlands, 1995.

[8] J.-C. Delvenne and V. D. Blondel, "Complexity of control on finite automata," Institute of Electrical and Electronics Engineers Transactions on Automatic Control, vol. 51, no. 6, pp. 977-986, 2006.

[9] J. Lygeros, C. Tomlin, and S. Sastry, "Controllers for reachability specifications for hybrid systems," Automatica, vol. 35, no. 3, pp. 349-370, 1999.

[10] X. Xu and Y. Hong, "Matrix approach to model matching of asynchronous sequential machines," Institute of Electrical and Electronics Engineers Transactions on Automatic Control, vol. 58, no. 11, pp. 2974-2979, 2013.

[11] E. Jimenez, J. Julvez, L. Recalde, and M. Silva, "On controllability of timed continuous petri net systems: the join free case," in Proceedings of the 44th IEEE Conference On Decision and Control, Seville, Spain, 2005.
[12] J. Júlvez, E. Jiménez, L. Recalde, and M. Silva, “On observability and design of observers in timed continuous petri net systems," IEEE Transactions on Automation Science and Engineering, vol. 5, no. 3, pp. 532-537, 2008.

[13] J. Latorre, E. Jimenez, J. Julvez, and M. Perez, "Macroreachability tree exploration for D.E.S design optimization," in Proceedings of the 6th EUROSIM Congress on Modelling and Simulation, Ljubljana, Slovenia, 2007.

[14] J. Latorre, E. Jimenez, J. Julvez, and M. Perez, "Control of discrete event systems modelled by petri nets," in Proceedings of the 7th EUROSIM Congress on Modelling and Simulation, Prague, Czech Republic, 2008.

[15] X. Han, Z. Chen, K. Zhang, Z. Liu, and Q. Zhang, "Modeling, reachability and controllability of bounded petri nets based on semi-tensor product of matrices," Asian Journal of Control, 2019.

[16] E. Vidal and F. Casacuberta, "Probabilistic finite-state machines-Part I," IEEE Transactions on Pattern Analysis and Machine Intelligence, vol. 27, no. 7, pp. 1013-1025, 2005.

[17] E. Vidal and F. Casacuberta, "Probabilistic finite-state machines-Part II," IEEE Transactions on Pattern Analysis and Machine Intelligence, vol. 27, no. 7, pp. 1026-1039, 2005.

[18] Z. Zhang, Z. Chen, and Z. Liu, "Modelling and reachability of probabilistic finite automata based on the semi-tensor product of matrices," Science China Information Sciences, vol. 61, no. 12, pp. 129-202, 2018.

[19] S. Shu, F. Lin, H. Ying, and X. Chen, "State estimation and detectability of probabilistic discrete event systems," Automatica, vol. 44, no. 12, pp. 3054-3060, 2008.

[20] Y. Li, F. Lin, and Z. H. Lin, "Supervisory control of probabilistic discrete-event systems with recovery," Institute of Electrical and Electronics Engineers Transactions on Automatic Control, vol. 44, no. 10, pp. 1971-1975, 1999.

[21] D. Z. Cheng, H. S. Qi, and Y. Zhao, An Introduction to SemiTensor Product of Matrices and Its Applications, World Scientific Publishing, Singapore, 2012.

[22] X. G. Han, Z. Q. Chen, and Z. X. Liu, "STP-based judgment method of reversibility and liveness of bounded Petri nets," Journal of Systems Science and Mathematical Sciences, vol. 36, pp. 361-370, 2016.

[23] Y. Guo, P. Wang, W. Gui, and C. Yang, "Set stability and set stabilization of Boolean control networks based on invariant subsets," Automatica, vol. 61, pp. 106-112, 2015.

[24] F. Li, H. Li, L. Xie, and Q. Zhou, "On stabilization and set stabilization of multivalued logical systems," Automatica, vol. 80, pp. 41-47, 2017.

[25] Y. Zou and J. Zhu, "Kalman decomposition for Boolean control networks," Automatica, vol. 54, pp. 65-71, 2015.

[26] D. Cheng, H. Qi, and Z. Liu, "From STP to game-based control," Science China Information Sciences, vol. 61, no. 1, Article ID 010201, 2018.

[27] H. Li and Y. Wang, "Lyapunov-based stability and construction of Lyapunov functions for Boolean networks," SIAM Journal on Control and Optimization, vol. 55, no. 6, pp. 3437-3457, 2017.

[28] X. Shen, Y. Wu, and T. Shen, "Logical control scheme with realtime statistical learning for residual gas fraction in IC engines," Science China Information Sciences, vol. 61, no. 1, Article ID 010203, 2018.

[29] H. Chen, X. Li, and J. Sun, "Stabilization, controllability and optimal control of Boolean networks with impulsive effects and state constraints," Institute of Electrical and Electronics Engineers Transactions on Automatic Control, vol. 60, no. 3, pp. 806-811, 2015. 
[30] H. Li and Y. Wang, "Further results on feedback stabilization control design of Boolean control networks," Automatica, vol. 83, pp. 303-308, 2017.

[31] Y. Li, H. Li, and W. Sun, "Event-triggered control for robust set stabilization of logical control networks," Automatica, vol. 95, pp. 556-560, 2018.

[32] J. Lu, H. Li, Y. Liu, and F. Li, "Survey on semi-tensor product method with its applications in logical networks and other finite-valued systems," IET Control Theory \& Applications, vol. 11, no. 13, pp. 2040-2047, 2017.

[33] M. Meng, L. Liu, and G. Feng, "Stability and 11 gain analysis of Boolean networks with Markovian jump parameters," Institute of Electrical and Electronics Engineers Transactions on Automatic Control, vol. 62, no. 8, pp. 4222-4228, 2017.

[34] Y. Zheng, H. Li, X. Ding, and Y. Liu, "Stabilization and set stabilization of delayed Boolean control networks based on trajectory stabilization," Journal of The Franklin Institute, vol. 354, no. 17, pp. 7812-7827, 2017.

[35] J. Zhong, J. Lu, T. Huang, and D. W. C. Ho, "Controllability and synchronization analysis of identical-hierarchy mixed-valued logical control networks," IEEE Transactions on Cybernetics, vol. 47, no. 11, pp. 3482-3493, 2017. 


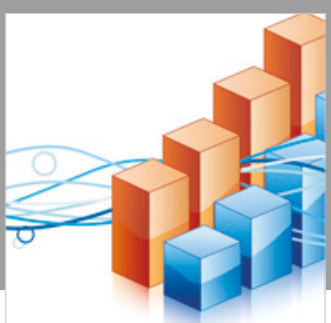

Advances in

Operations Research

\section{-n-m}
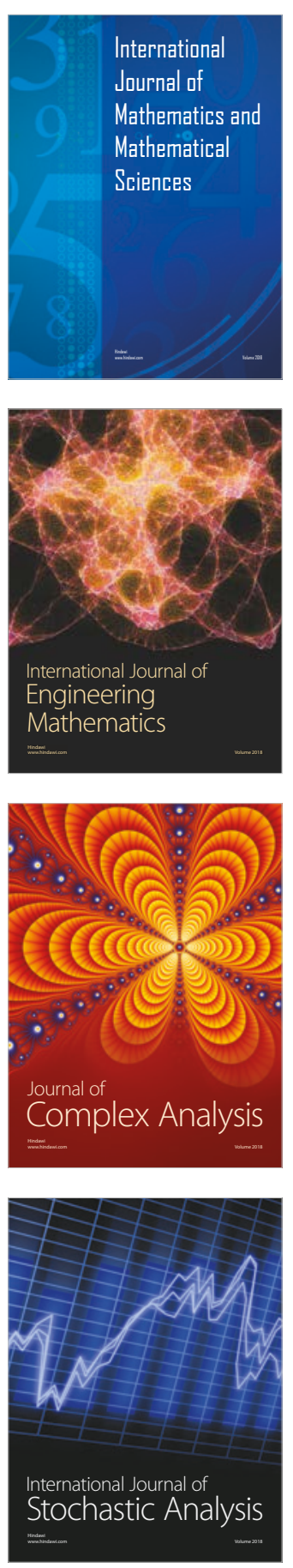
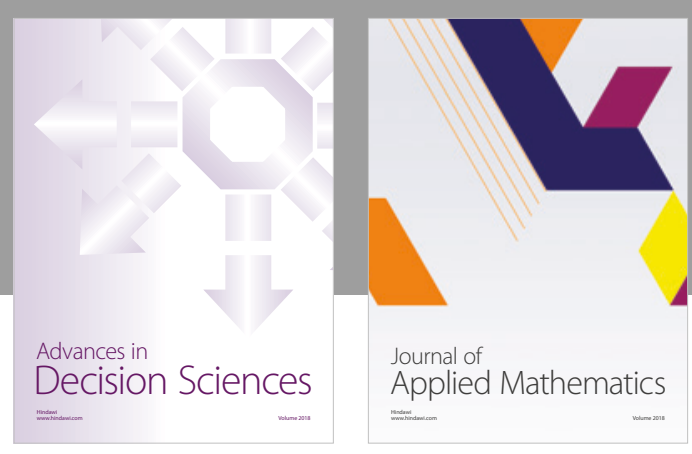

Journal of

Applied Mathematics
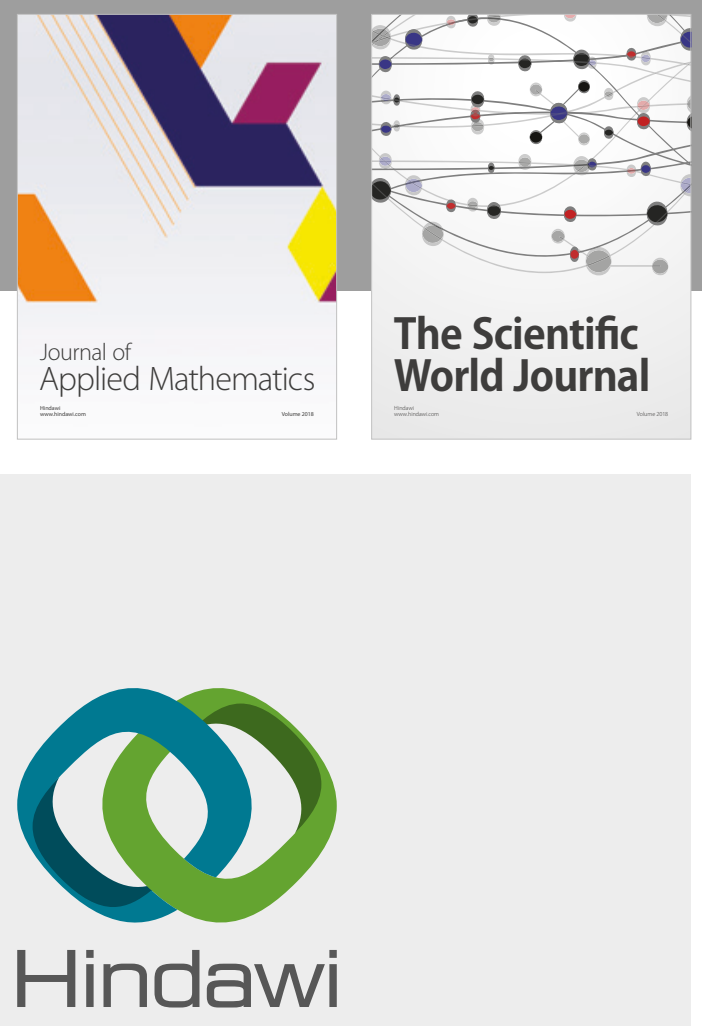

Submit your manuscripts at

www.hindawi.com

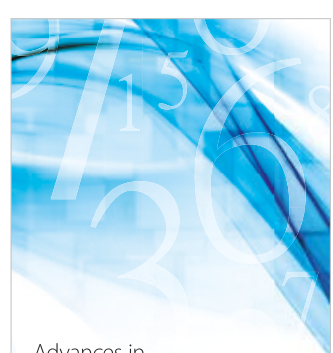

Advances in
Numerical Analysis
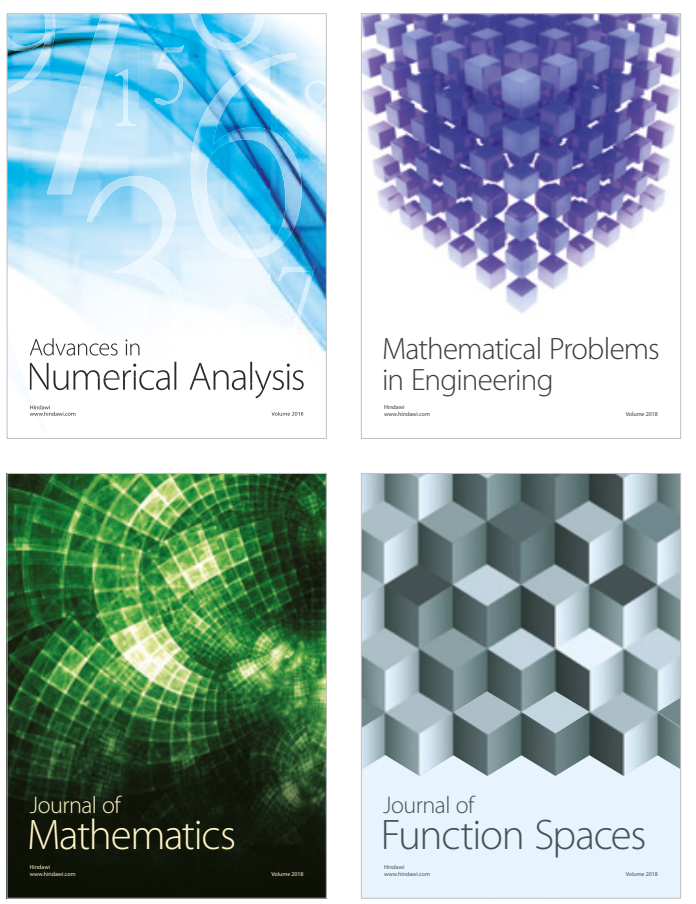

Mathematical Problems in Engineering

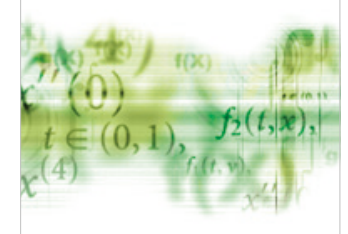

International Journal of

Differential Equations

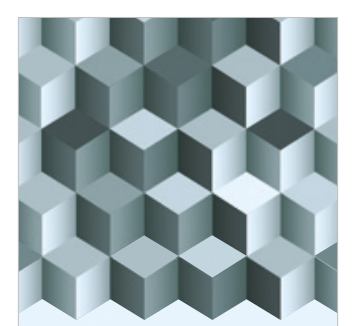

Journal of

Function Spaces

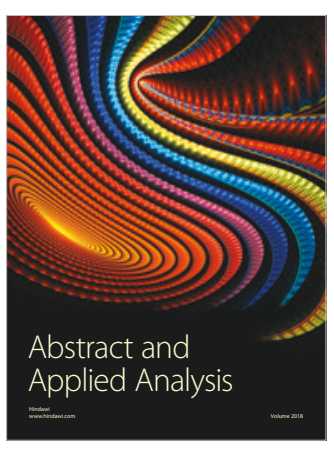

The Scientific

World Journal

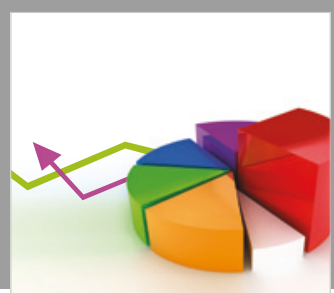

Journal of

Probability and Statistics
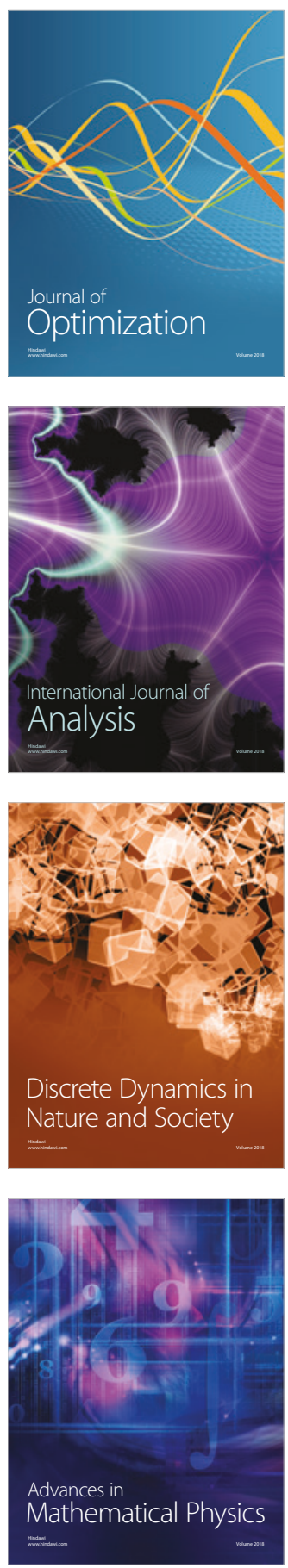